University of Nebraska - Lincoln

DigitalCommons@University of Nebraska - Lincoln

August 2003

\title{
Elastic wave propagation and scattering in solids with uniaxially aligned cracks
}

Liyong Yang

University of Nebraska - Lincoln, lyang4@unl.edu

Joseph A. Turner

University of Nebraska - Lincoln, jaturner@unl.edu

Follow this and additional works at: https://digitalcommons.unl.edu/cmrafacpub

Part of the Nanoscience and Nanotechnology Commons

Yang, Liyong and Turner, Joseph A., "Elastic wave propagation and scattering in solids with uniaxially aligned cracks" (2003). Faculty Publications from Nebraska Center for Materials and Nanoscience. 61. https://digitalcommons.unl.edu/cmrafacpub/61

This Article is brought to you for free and open access by the Materials and Nanoscience, Nebraska Center for (NCMN) at DigitalCommons@University of Nebraska - Lincoln. It has been accepted for inclusion in Faculty Publications from Nebraska Center for Materials and Nanoscience by an authorized administrator of DigitalCommons@University of Nebraska - Lincoln. 


\title{
Elastic wave propagation and scattering in solids with uniaxially aligned cracks
}

\author{
Liyong Yang and Joseph A. Turner \\ Department of Engineering Mechanics, University of Nebraska-Lincoln, W317.4 Nebraska Hall, Lincoln, \\ Nebraska 68588-0526
}

(Received 11 November 2002; revised 18 May 2003; accepted 19 May 2003)

\begin{abstract}
In this article, elastic wave propagation and scattering in a solid medium permeated by uniaxially aligned penny-shaped microcracks are studied. The crack alignment refers to the case in which the unit normals of all cracks are randomly oriented within a plane of isotropy. The analysis is restricted to the limit of the noninteraction approximation among individual cracks. Explicit expressions for attenuations and wave speeds of the shear horizontal, quasilongitudinal, and quasishear vertical waves are obtained using stochastic wave theory in a generalized dyadic approach. The ensemble average elastic wave response is governed by the Dyson equation, which is solved in terms of the anisotropic elastic Green's dyadic. The analysis of expressions is limited to frequencies below the geometric optics limit. The resulting attenuations are investigated in terms of the directional, frequency, and damage dependence. In particular, the attenuations are simplified considerably within the low frequency Rayleigh regime. Finally, numerical results are presented and discussed in terms of the relevant dependent parameters. (c) 2003 Acoustical Society of America.
\end{abstract}

[DOI: $10.1121 / 1.1592158]$

PACS numbers: 43.20.Bi, 43.20.Gp, 43.35.Cg [DEC]

\section{INTRODUCTION}

The investigation of wave propagation and scattering of elastic waves in damaged solids is of considerable interest to nondestructive evaluation and materials characterization, particularly for ultrasonic techniques. Analytical and experimental examinations of attenuation and wave speeds of ultrasonic waves in cracked solids provide a direct approach for the detection of material damage. Material responses, which are typically evaluated ultrasonically by the decrease in wave velocity or increase in wave attenuation, vary with microcracking changes. Both of these phenomena are caused by the stiffness degradation of the material by the cracks. Studies of elastic wave attenuation in cracked solid media have been studied for at least 30 years, since the work of Mal, ${ }^{1,2}$ Piau, ${ }^{3}$ Chatterjee et al.,${ }^{4}$ Hudson, ${ }^{5}$ Martin, ${ }^{6}$ Krenk, ${ }^{7}$ and Martin and Wickham. ${ }^{8}$ In addition, wave propagation in cracked solids has been reviewed by Zhang and Achenbach, ${ }^{9}$ Zhang and Gross, ${ }^{10,11}$ Smyshlyaev and Willis, ${ }^{12}$ and Eriksson and Datta. ${ }^{13}$ Previous research has primarily been focused on specific wave types and wave directions for either a single crack or distributed cracks, but a more comprehensive, general study has never been undertaken. In our previous article, ${ }^{14}$ explicit general expressions of wave attenuations and wave speeds in a medium with damage from randomly distributed penny-shaped microcracks were derived. Under the assumption of statistical isotropy used in that work, the attenuation is independent of propagation direction. However, in the case of structural materials such as concrete, polycrystalline metals, fiber-reinforced composites, and others, those microcracks induced by directional loading or temperature are typically parallel to some direction. In this case, the effective media may acquire an anisotropy essentially due to the presence of such uniaxially aligned cracks. Thus, the scattering attenuation is a function of propagation direc- tion. The analysis of this scattering attenuation is, therefore, more complicated than that of the isotropic case.

In this article, the framework used previously ${ }^{14}$ is extended to study the attenuation of elastic waves in solids with uniaxially aligned cracks that are statistically homogeneous. Again, the microcracks are assumed to be noninteracting, penny-shaped cracks. Here, the unit normals of all cracks are assumed to be coplanar, but random within this plane of isotropy. Thus, the uniaxial symmetry direction is perpendicular to this plane. This case is different from the case of perfect crack alignment by all cracks, as discussed by Hudson, ${ }^{5}$ for example. The effective elastic moduli of the medium that contains many penny-shaped cracks are reviewed by Nemat-Nasser, ${ }^{15}$ Kachanov, ${ }^{16}$ Krajcinovic, ${ }^{17}$ and others. General wave propagation and scattering problems for anisotropic media are discussed by Stanke and Kino, ${ }^{18}$ Ahmed and Thompson, ${ }^{19}$ and Hirsekorn. ${ }^{20,21}$ All those discussions involve a scattering integral with an isotropic Green's function. The use of an anisotropic Green's function for modeling the scattering in anisotropic media was investigated by Turner. ${ }^{22}$ Here, this approach is employed as well to formulate the uniaxially aligned crack problem. In this way, the mean response is written in terms of the Dyson equation as discussed by Frisch ${ }^{23}$ and Weaver. ${ }^{24}$ The Dyson equation is solved in the spatial Fourier transform domain within the limits of the first-order smoothing approximation (FOSA), or Keller ${ }^{25}$ approximation. A further approximation is also made which restricts the results to frequencies below the high-frequency geometric optics limit. The resulting attenuations are shown to be directional dependent, frequency dependent, and damage dependent for the shear horizontal, quasilongitudinal, and quasishear vertical waves. In particular, the angular dependence of the attenuations in the Rayleigh limit is obtained explicitly. Outside the Rayleigh limit, 
simple expressions of the attenuations of the shear horizontal, quasilongitudinal, and quasishear vertical waves are derived in terms of integrations on the unit circle. Quantitative and qualitative comparisons with previous results by Zhang and Gross, ${ }^{10,11}$ Zhang and Achenbach, ${ }^{9}$ Eriksson and Datta, ${ }^{13}$ Ahmed and Thompson, ${ }^{19}$ and Turner $^{22}$ show that the more general, direct expressions derived here are reliable and comprehensive for practical applications of detecting damage from microcracks.

This article is organized in the following manner. The effective elastic properties of a solid containing uniaxially aligned cracks are discussed in Sec. II. The wave propagation and scattering model described by an anisotropic Green's function is included for completeness in Sec. III. The attenuations of each wave type through the uniaxially aligned cracks are given in a direct form subsequently in Sec. IV. In Sec. V, numerical results and discussion are presented. Finally, conclusions are presented in Sec. VI.

\section{EFFECTIVE PROPERTIES IN SOLIDS WITH UNIAXIALLY ALIGNED CRACKS}

The effective stiffness attributed to a single pennyshaped crack, which is located within an infinite, homogeneous, isotropic and elastic continuum is considered first. Estimating the effective elastic properties of a statistically homogeneous elastic solid which contains a large number of microcracks was investigated by Nemat-Nasser and Hori, ${ }^{15}$ Kachanov, ${ }^{16}$ and others. Using similar methods, the effective stiffness attributable to a single, penny-shaped crack of radius $a$ in a unit volume, called the crack basis Green's function, is given in the form ${ }^{14}$

$$
\mathbf{C}^{(s)}=2 \mu d_{i} \mathbf{I}^{i},
$$

where the repeated index $i$ denotes the summation convention over the range of $i=1-6$. It should be noted that this summation convention and range are used throughout this article. The coefficients $d_{i}$ are given by

$d_{1}=0, \quad d_{2}=\frac{16}{3} \frac{\nu^{2}(1-\nu)}{(1-2 \nu)^{2}} a^{3}, \quad d_{3}=d_{4}=\frac{16}{3} \frac{\nu(1-\nu)}{1-2 \nu} a^{3}$,

$d_{5}=\frac{32}{3} \frac{1-\nu}{2-\nu} a^{3}, \quad d_{6}=-\frac{16}{3} \frac{\nu(1-\nu)}{2-\nu} a^{3}$.

The stiffness of a single crack is dependent on the unit normal $\hat{\mathbf{m}}$, which defines the crack orientation. This orientation is implicit in the tensors $\mathbf{I}$. These basis tensors are given in terms of unit vector $\hat{\mathbf{m}}$ and Kronecker delta function as follows. ${ }^{26}$

$$
\begin{aligned}
& I_{i j m n}^{1}=\frac{1}{2}\left(\delta_{i m} \delta_{j n}+\delta_{i n} \delta_{j m}\right), \quad I_{i j m n}^{2}=\delta_{i j} \delta_{m n}, \\
& I_{i j m n}^{3}=\delta_{i j} \hat{m}_{m} \hat{m}_{n}, \quad I_{i j m n}^{4}=\hat{m}_{i} \hat{m}_{j} \delta_{m n}, \\
& I_{i j m n}^{6}=\hat{m}_{i} \hat{m}_{j} \hat{m}_{m} \hat{m}_{n}, \\
& I_{i j m n}^{5}=\frac{1}{4}\left(\hat{m}_{i} \hat{m}_{m} \delta_{j n}+\hat{m}_{i} \hat{m}_{n} \delta_{j m}+\hat{m}_{j} \hat{m}_{m} \delta_{i n}+\hat{m}_{j} \hat{m}_{n} \delta_{i m}\right) .
\end{aligned}
$$

The ensemble average properties contributed by all cracks are considered next. The cracks are assumed to be embedded in an infinitely extended, homogeneous, isotropic and elastic three-dimensional continuum. The penny-shaped

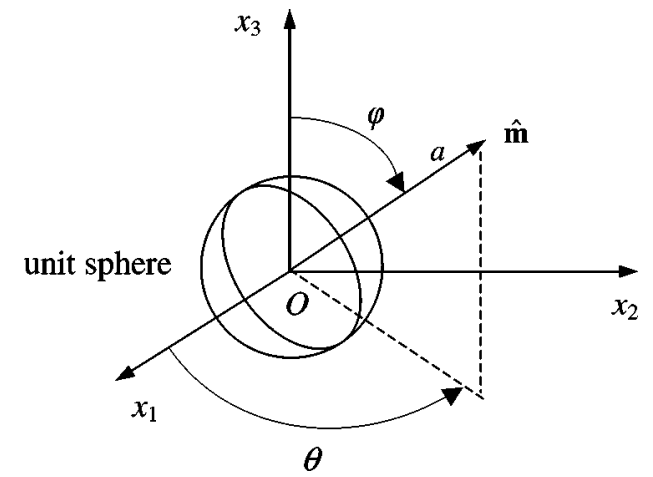

FIG. 1. Geometry of a penny-shaped crack.

crack is characterized by its radius $a$ and two Euler angles $\theta$ and $\varphi$, which define the direction of the unit normal $\hat{\mathbf{m}}$ as shown in Fig. 1. It is also assumed that the microcracks do not interact with each other. Thus, the effective stiffness may be determined by integration over a continuous distribution of crack sizes and orientations. In general, the specific distribution of the crack radii and orientations is described by the probability density function $W(a, \theta, \varphi)$. In some cases, the microcrack radii and orientations are often correlated. Here, it is assumed that the microcrack radii and orientations are not correlated. As such, the density function may be separated into independent radius and orientation functions of the form

$$
W(a, \theta, \varphi)=A(a) \zeta(\theta, \varphi) .
$$

It is also assumed that all microcracks are parallel to the $x_{3}$ axis ( $\hat{\mathbf{n}}$ direction) with their unit normals (lying in the $x_{1}-x_{2}$ plane), having a random distribution, as shown in Fig. 2. In this situation, due to the symmetry about the $x_{1}$ $-x_{2}$ plane, the average elastic properties are those of transverse isotropy, with the $x_{3}$ axis as the uniaxial symmetry axis. Here, the distribution of the microcracks is supposed to be dilute, and the distribution of the crack sizes is also assumed to be independent of their orientations. The crack orientation distribution function in Eq. (4), which implies that the orientation function $\zeta(\theta, \varphi)$ is independent of the angle $\theta$, is then given by

$$
\zeta(\theta, \varphi)=2 \delta\left(\varphi-\frac{\pi}{2}\right)
$$

Therefore, the effective continuum material properties caused by all microcracks per unit volume are weighted by
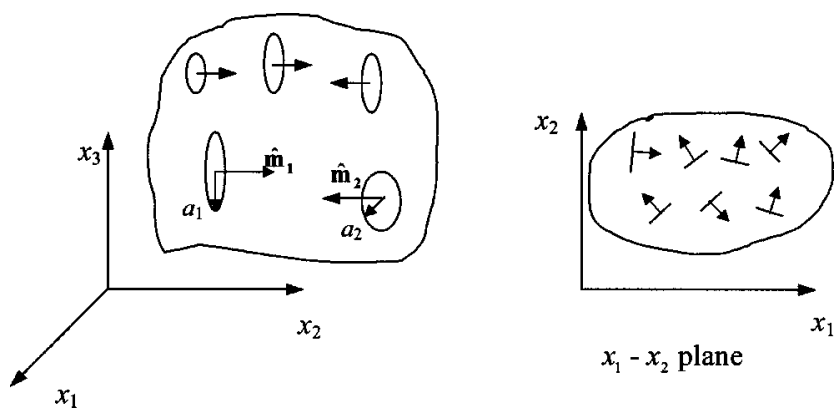

FIG. 2. The distribution of microcracks parallel to the $x_{3}$-axis. 
the density function, Eq. (4), over the basis Green's function, Eq. (1), and are then given by

$C_{i j m n}^{*}=\frac{\varepsilon}{2 \pi} \int_{0}^{2 \pi} \int_{-\pi / 2}^{\pi / 2} \delta\left(\varphi-\frac{\pi}{2}\right) C_{i j m n}^{(s)}(\theta, \varphi) \sin \varphi d \theta d \varphi$.

In Eq. (6), the nondimensional microcrack density per unit volume is defined by

$$
\varepsilon=N\left\langle a^{3}\right\rangle=\int_{a^{-}}^{a^{+}} A(a) a^{3} d a,
$$

where $N$ is number of cracks per unit volume and the angular brackets denote the ensemble average. This damage density definition was introduced first by $\mathrm{Walsh}^{27}$ for the case of a statistically isotropic distribution of penny-shaped microcracks. A more general form of the damage density is discussed by Budiansky. ${ }^{28}$ The basis function $C_{i j m n}^{(s)}$ is expressed in Eq. (1). By integrating over the Euler angles in Eq. (6), the effective stiffness due to the distribution of uniaxially aligned penny-shaped microcracks is derived as

$$
C_{i j m n}^{*}=D a_{i} \mathbf{I}^{i}
$$

where the coefficients $D$ and $a_{i}$ are

$$
\begin{aligned}
& D=2 \varepsilon \mu \frac{16}{3} \frac{1-\nu}{2-\nu}, \quad a_{1}=1-\frac{\nu}{4}, \\
& a_{2}=\frac{\nu\left(15-20 \nu+4 \nu^{2}\right)}{8(1-2 \nu)^{2}}, \quad a_{3}=a_{4}=\frac{\nu(-7+2 \nu)}{8(1-2 \nu)}, \\
& a_{5}=1-\frac{\nu}{2}, \quad a_{6}=-\frac{3 \nu}{8} .
\end{aligned}
$$

In the tensors I used in Eq. (8), the orientation is that of the symmetry direction $\hat{\mathbf{n}}$, rather than the direction $\hat{\mathbf{m}}$. It is hoped that this notation is not confusing to the reader. If the original undamaged state of the material is homogeneous and isotropic, the stiffness tensor is given in the standard form $\mathbf{C}^{0}=\lambda \mathbf{I}^{2}+2 \mu \mathbf{I}^{1}$.

The ensemble effective stiffness is now redefined such that the average fluctuations are zero as done previously. ${ }^{14}$ Such a procedure, while not necessary, is convenient for the calculation of material covariance and attenuation. The moduli are assumed to be spatially varying and of the form

$$
\overline{\mathbf{C}}(\mathbf{x})=\overline{\mathbf{C}}^{0}+\delta \overline{\mathbf{C}}(\mathbf{x}),
$$

where

$$
\overline{\mathbf{C}}^{0}=\mathbf{C}^{0}-\mathbf{C}^{*} .
$$

Thus, the average moduli have the form

$$
\overline{\mathbf{C}}^{0}=\langle\overline{\mathbf{C}}(\mathbf{x})\rangle=\lambda_{\perp} \mathbf{I}^{2}+\mu_{\perp} \mathbf{I}^{1}+\Gamma_{1}\left(\mathbf{I}^{3}+\mathbf{I}^{4}\right)+\Gamma_{2} \mathbf{I}^{5}+\Gamma_{3} \mathbf{I}^{6},
$$

where the effective elastic constants are

$$
\begin{gathered}
\lambda_{\perp}=\lambda-\frac{D \nu\left(15-20 \nu+4 \nu^{2}\right)}{8(1-2 \nu)^{2}}, \quad \mu_{\perp}=\mu-\frac{D(4-\nu)}{8}, \\
\Gamma_{1}=\frac{D \nu(7-2 \nu)}{8(1-2 \nu)}, \quad \Gamma_{2}=D\left(-1+\frac{\nu}{2}\right), \quad \Gamma_{3}=\frac{3 D \nu}{8} .
\end{gathered}
$$

The moduli fluctuations, which have zero mean, $\langle\delta \bar{C}\rangle=0$, are given by

$$
\delta \overline{\mathbf{C}}=\mathbf{C}^{*}-\mathbf{C}^{(s)} H(\mathbf{x}),
$$

and the function $H(\mathbf{x})$ is defined as

$$
H(\mathbf{x})=\left\{\begin{array}{ll}
1 & \text { if } \mathbf{x} \in \mathbf{S} \\
0 & \text { otherwise }
\end{array},\right.
$$

where $\mathbf{S}$ denotes the space of all cracks.

In the next section, the fundamental elastodynamics of elastic wave propagation and scattering are introduced in terms of appropriate Green's dyadic. The formalism is developed for a transversely isotropic material. The mean response, which is expressed in terms of the Dyson equation, is discussed for the case of a transversely isotropic medium. The elastic modulus tensor is specified for this case and expressions of the attenuation for each wave type are given.

\section{ELASTIC WAVE PROPAGATION AND SCATTERING MODEL}

The equation of motion for the elastodynamic response of an infinite, linear-elastic material to deformation is given in terms of the Green's dyadic by

$$
\begin{aligned}
& \left\{-\delta_{j m} \rho(\mathbf{x}) \partial_{t}^{2}+\partial x_{i} \bar{C}_{i j m n}(\mathbf{x}) \partial x_{n}\right\} G_{m \alpha}\left(\mathbf{x}, \mathbf{x}^{\prime} ; t\right) \\
& =\delta_{j \alpha} \delta^{3}\left(\mathbf{x}-\mathbf{x}^{\prime}\right) \delta(t)
\end{aligned}
$$

where $\delta^{3}\left(\mathbf{x}-\mathbf{x}^{\prime}\right)$ is the three-dimensional spatial delta function. The second order Green's dyadic, $G_{m \alpha}\left(\mathbf{x}, \mathbf{x}^{\prime} ; t\right)$, defines the response at location $\mathbf{x}$ in the $m$ th direction to a unit impulse at location $\mathbf{x}^{\prime}$ in the $\alpha$ th direction. The moduli are considered to vary spatially and density is assumed uniform throughout with units chosen such that density is unity. The moduli $\overline{\mathbf{C}}$ are assumed to be spatially heterogeneous and have the form in Eq. (10). The covariance of the moduli is characterized by an eighth-rank tensor

$$
\left\langle\delta C_{i j m n}(\mathbf{x}) \delta C_{\alpha \beta \gamma \delta}(\mathbf{y})\right\rangle=\Xi_{i j m n}^{\alpha \beta \gamma \delta} W(\mathbf{x}-\mathbf{y}) .
$$

The spatial and tensorial parts of the above covariance, $\Xi$ and $W$, respectively, are assumed independent. The spatial correlation function $W$ is also assumed a function of the difference between two vectors, $\mathbf{x}-\mathbf{y}$. This assumption implies that the medium is statistically homogeneous. However, the additional assumption of statistical isotropy as considered previously, ${ }^{14}$ such that $W$ is a function of $|\mathbf{x}-\mathbf{y}|$, is not made here.

The mean response, $\langle\mathbf{G}\rangle$, is governed by the Dyson equation which is given by ${ }^{23,24}$

$$
\begin{aligned}
\left\langle G_{i \alpha}\left(\mathbf{x}, \mathbf{x}^{\prime}\right)\right\rangle= & G_{i \alpha}^{0}\left(\mathbf{x}, \mathbf{x}^{\prime}\right)+\iint G_{i \beta}^{0}(\mathbf{x}, \mathbf{y}) M_{\beta j}(\mathbf{y}, \mathbf{z}) \\
& \times\left\langle G_{j \alpha}\left(\mathbf{z}, \mathbf{x}^{\prime}\right)\right\rangle d^{3} y d^{3} z .
\end{aligned}
$$

In Eq. (18), the quantity $\mathbf{G}^{0}$ is the bare Green's dyadic. It defines the response of the medium without heterogeneities, namely, the solution of Eq. (16) with $\delta \bar{C}_{i j m n}(\mathbf{x})=0$. The second order tensor $\mathbf{M}$ is the mass or self-energy operator. The Dyson equation, Eq. (18), is easily solved in Fourier trans- 
form space under the assumption of statistical homogeneity. The assumption of statistical homogeneity ensures that $\mathbf{G}^{0}$, $\mathbf{M}$ and $\langle\mathbf{G}\rangle$ are functions of a single wave vector in Fourier space. The Dyson equation is then transformed and solved to give the result for $\langle\mathbf{G}(\mathbf{p})\rangle$ of the form

$$
\langle\mathbf{G}(\mathbf{p})\rangle=\left[\mathbf{G}^{0}(\mathbf{p})^{-1}-\tilde{\mathbf{M}}(\mathbf{p})\right]^{-1} .
$$

Here, $\widetilde{\mathbf{M}}$ is the spatial transform of the self-energy. An approximation of the self-energy $\mathbf{M}$ can be written as an expansion in powers of moduli fluctuations. To first order, ${ }^{23,25}$ $\mathbf{M}$ is expressed $\mathrm{as}^{24}$

$$
\begin{aligned}
& M_{\beta j}(\mathbf{y}, \mathbf{z}) \\
& \quad \approx\left\langle\frac{\partial}{\partial y_{\alpha}} \delta C_{\alpha \beta \gamma \delta}(\mathbf{y}) \frac{\partial}{\partial y_{\delta}} G_{\gamma m}^{0}(\mathbf{y}, \mathbf{z}) \frac{\partial}{\partial z_{i}} \delta C_{i j m n}(\mathbf{z}) \frac{\partial}{\partial z_{n}}\right\rangle .
\end{aligned}
$$

Such an approximation is assumed valid if the fluctuations, $\delta \mathbf{C}$, are not too large. The components of $\tilde{\mathbf{M}}$, which are employed to calculate the phase velocity and attenuation of the wave modes, are discussed next. Further details of the scattering theory can be reviewed by the reader in the articles of Karal and Keller, ${ }^{25}$ Frisch, ${ }^{23}$ Stanke and Kino, ${ }^{18}$ Weaver, ${ }^{24}$ and Turner. ${ }^{22}$

The medium of uniaxially aligned cracks is considered to be transversely isotropic, a medium with a single symmetry axis defined here by the unit vector $\hat{\mathbf{n}}$. The fourth-rank elastic moduli tensor, $\overline{\mathbf{C}}$, in a transversely isotropic medium, given in Eq. (12), is written in terms of $\hat{\mathbf{n}}$ by

$$
\begin{aligned}
\bar{C}_{i j m n}= & \lambda_{\perp} \delta_{i j} \delta_{m n}+\mu_{\perp}\left(\delta_{i m} \delta_{j n}+\delta_{i n} \delta_{j m}\right)+\Gamma_{1}\left(\delta_{i j} \hat{n}_{m} \hat{n}_{n}\right. \\
& \left.+\delta_{m n} \hat{n}_{i} \hat{n}_{j}\right)+\Gamma_{2}\left(\delta_{i m} \hat{n}_{j} \hat{n}_{n}+\delta_{i n} \hat{n}_{j} \hat{n}_{m}+\delta_{j m} \hat{n}_{i} \hat{n}_{n}\right. \\
& \left.+\delta_{j n} \hat{n}_{i} \hat{n}_{m}\right)+\Gamma_{3} \hat{n}_{i} \hat{n}_{j} \hat{n}_{m} \hat{n}_{n} .
\end{aligned}
$$

The above elastic constants are defined in Eqs. (13).

For propagation in the $\hat{\mathbf{p}}$ direction, the shear horizontal wave $(S H)$ in a transversely isotropic medium is polarized in direction $\hat{\mathbf{u}}_{1}$, that is perpendicular to the plane defined by $\hat{\mathbf{p}}$ and $\hat{\mathbf{n}}$. The angle between the $\hat{\mathbf{p}}$ and $\hat{\mathbf{n}}$ is defined as $\Theta$. The quasi- $P$ and quasi- $S V$ waves are polarized in directions defined by $\hat{\mathbf{u}}_{2}$ and $\hat{\mathbf{u}}_{3}$, respectively, both of which lie in the $\hat{\mathbf{p}}$ $-\hat{\mathbf{n}}$ plane. It is noted that $\hat{\mathbf{u}}_{1}, \hat{\mathbf{u}}_{2}$, and $\hat{\mathbf{u}}_{3}$ form an orthonormal basis such that $\hat{\mathbf{u}}_{3}=\hat{\mathbf{u}}_{1} \times \hat{\mathbf{u}}_{2}$. The vector $\hat{\mathbf{u}}_{2}$ is directed at an angle $\psi$ from the propagation direction $\hat{\mathbf{p}}$ (see Fig. 3). $\mathbf{G}^{0}$ may be diagonalized by using the directions $\hat{\mathbf{u}}_{2}$ and $\hat{\mathbf{u}}_{3}$, such that $\mathbf{I}-\hat{\mathbf{u}}_{1} \hat{\mathbf{u}}_{1}=\hat{\mathbf{u}}_{2} \hat{\mathbf{u}}_{2}+\hat{\mathbf{u}}_{3} \hat{\mathbf{u}}_{3}$.

Substituting the elastic stiffness tensor $\overline{\mathbf{C}}$ into the transform form of the equation of motion, Eq. (16), gives in direct notation $^{22}$

$$
\begin{aligned}
\left\{\hat{\mathbf{u}}_{1}\right. & \hat{\mathbf{u}}_{1}\left[\omega^{2}-p^{2}\left(\mu_{\perp}+\Gamma_{2} \cos ^{2} \Theta\right)\right]+\hat{\mathbf{u}}_{2} \hat{\mathbf{u}}_{2}\left[\omega^{2}\right. \\
& \left.-p^{2}\left(Q+P \cos ^{2} \psi+R \cos ^{2}(\Theta+\psi)\right)\right]+\hat{\mathbf{u}}_{3} \hat{\mathbf{u}}_{3}\left[\omega^{2}\right. \\
& \left.\left.-p^{2}\left(Q+P \sin ^{2} \psi+R \sin ^{2}(\Theta+\psi)\right)\right]\right\} \cdot \mathbf{G}^{0}(\mathbf{p})=\mathbf{I} .
\end{aligned}
$$

The quantities $Q, P$, and $R$ in Eq. (22) are defined by

$$
\begin{aligned}
& Q=\mu_{\perp}+\Gamma_{2}(\hat{\mathbf{p}} \cdot \hat{\mathbf{n}})^{2}-\left(\Gamma_{1}+\Gamma_{2}\right)\left(1-(\hat{\mathbf{p}} \cdot \hat{\mathbf{n}})^{2}\right), \\
& P=\lambda_{\perp}+\mu_{\perp}+\Gamma_{1}+\Gamma_{2},
\end{aligned}
$$

$$
R=\Gamma_{1}+2 \Gamma_{2}+\Gamma_{3}(\hat{\mathbf{p}} \cdot \hat{\mathbf{n}})^{2} .
$$

It should also be kept in mind that the vectors $\hat{\mathbf{u}}_{2}$ and $\hat{\mathbf{u}}_{3}$ are functions of the direction of propagation, $\hat{\mathbf{p}}$, relative to the uniaxial symmetry direction, $\hat{\mathbf{n}}$. This dependence, $\psi$ $=\psi(\Theta)$ will remain implicit throughout.

The bare Green's dyadic is then presented in the form

$$
\mathbf{G}^{0}(\mathbf{p})=g_{S H}^{0}(\mathbf{p}) \hat{\mathbf{u}}_{1} \hat{\mathbf{u}}_{1}+g_{q P}^{0}(\mathbf{p}) \hat{\mathbf{u}}_{2} \hat{\mathbf{u}}_{2}+g_{q S V}^{0}(\mathbf{p}) \hat{\mathbf{u}}_{3} \hat{\mathbf{u}}_{3},
$$

where the dispersion relations for the bare response of the $S H, q P$, and $q S V$ waves are given by

$$
\begin{aligned}
g_{S H}^{0}(\mathbf{p}) & =\left[\omega^{2}-p^{2}\left(\mu_{\perp}+\Gamma_{2} \cos ^{2} \Theta\right)\right]^{-1} \\
& =\left[\omega^{2}-p^{2} c_{S H}^{2}\right]^{-1}, \\
g_{q P}^{0}(\mathbf{p}) & =\left[\omega^{2}-p^{2}\left(Q+P \cos ^{2} \psi+R \cos ^{2}(\Theta+\psi)\right)\right]^{-1} \\
& =\left[\omega^{2}-p^{2} c_{q P}^{2}\right]^{-1}, \\
g_{q S V}^{0}(\mathbf{p}) & =\left[\omega^{2}-p^{2}\left(Q+P \sin ^{2} \psi+R \sin ^{2}(\Theta+\psi)\right)\right]^{-1} \\
& =\left[\omega^{2}-p^{2} c_{q S V}^{2}\right]^{-1},
\end{aligned}
$$

with $Q, P$, and $R$ defined in Eqs. (23).

The mean response, $\langle\mathbf{G}(\mathbf{p})\rangle$, is governed by the Dyson equation, Eq. (19). The solution of $\langle\mathbf{G}(\mathbf{p})\rangle$ is expressed in terms of $\mathbf{G}^{0}(\mathbf{p})$ and $\tilde{\mathbf{M}}(\mathbf{p})$. Similar to $\mathbf{G}^{0}$, the mean response $\langle\mathbf{G}(\mathbf{p})\rangle$ and self-energy $\tilde{\mathbf{M}}(\mathbf{p})$ may be written in terms of the orthonormal basis defined by $\hat{\mathbf{u}}_{1}, \hat{\mathbf{u}}_{2}$, and $\hat{\mathbf{u}}_{3}$ in the form

$$
\begin{aligned}
& \langle\mathbf{G}(\mathbf{p})\rangle=g_{S H}(\mathbf{p}) \hat{\mathbf{u}}_{1} \hat{\mathbf{u}}_{1}+g_{q P}(\mathbf{p}) \hat{\mathbf{u}}_{2} \hat{\mathbf{u}}_{2}+g_{q S V}(\mathbf{p}) \hat{\mathbf{u}}_{3} \hat{\mathbf{u}}_{3}, \\
& \tilde{\mathbf{M}}(\mathbf{p})=m_{S H}(\mathbf{p}) \hat{\mathbf{u}}_{1} \hat{\mathbf{u}}_{1}+m_{q P}(\mathbf{p}) \hat{\mathbf{u}}_{2} \hat{\mathbf{u}}_{2}+m_{q S V}(\mathbf{p}) \hat{\mathbf{u}}_{3} \hat{\mathbf{u}}_{3},
\end{aligned}
$$

where it is again noted that the propagation direction $\hat{\mathbf{p}}$ is implicit within the directions $\hat{\mathbf{u}}_{1}, \hat{\mathbf{u}}_{2}$, and $\hat{\mathbf{u}}_{3}$.

The dispersion relations for the mean response are then given by the solution of the Dyson equation, Eq. (19), as

$g_{\beta}(\mathbf{p})=\left[g_{\beta}^{0}(\mathbf{p})^{-1}-m_{\beta}(\mathbf{p})\right]^{-1}=\left[\omega^{2}-p^{2} c_{\beta}^{2}-m_{\beta}(\mathbf{p})\right]^{-1}$,

for each wave type, $\beta$. These are the expressions for the dispersion relation of the mean response, which defines the phase velocity and attenuation of each wave type from solution of

$$
\omega^{2}-p^{2} c_{\beta}^{2}-m_{\beta}(\mathbf{p})=0,
$$

for the wave vector $\mathbf{p}$. The attenuation of each wave type is given by the imaginary part of p. Explicit expressions of the attenuation can be determined using an approximation valid below the high-frequency geometric optics limit $\left(m_{\beta}(\mathbf{p})\right.$ $\left.\approx m_{\beta}\left(\omega / c_{\beta} \hat{\mathbf{p}}\right)\right) .{ }^{18,24}$ This approximation allows the imaginary part of $\mathbf{p}$ to be calculated directly from Eq. (28). Thus, the attenuation of each wave type is given as

$$
\alpha_{\beta}(\hat{\mathbf{p}})=-\frac{1}{2 \omega c_{\beta}(\hat{\mathbf{p}})} \operatorname{Im} m_{\beta}\left(\frac{\omega}{c_{\beta}} \hat{\mathbf{p}}\right) .
$$

The attenuations for the three wave types, which are each defined in Eq. (29), are finally given in the general form $^{22}$

L. Yang and A. Turner: Elastic wave propagation and scattering 


$$
\begin{aligned}
& \alpha_{\beta}(\hat{\mathbf{p}})=\frac{1}{c_{\beta}^{3}(\hat{\mathbf{p}})}\left\{\frac{\pi}{4} \int d^{2} \hat{s} \frac{\omega^{4}}{c_{S H}^{5}(\hat{\mathbf{s}})} W\left(\frac{\omega}{c_{\beta}(\hat{\mathbf{p}})} \hat{\mathbf{p}}-\frac{\omega}{c_{S H}(\hat{\mathbf{s}})} \hat{\mathbf{s}}\right)\right. \\
& \times \widetilde{\Xi}_{\cdots \cdot \hat{\mathbf{u}}_{K} \hat{\mathbf{p}} \hat{\mathbf{v}}_{K} \hat{\mathbf{v}}_{1} \hat{\mathbf{v}}_{1}}+\frac{\pi}{4} \int d^{2} \hat{s} \frac{\omega^{4}}{c_{q P}^{5}(\hat{\mathbf{s}})} \\
& \times W\left(\frac{\omega}{c_{\beta}(\hat{\mathbf{p}})} \hat{\mathbf{p}}-\frac{\omega}{c_{q P}(\hat{\mathbf{s}})} \hat{\mathbf{s}}\right) \\
& \times \widetilde{\Xi}_{\cdots \cdots \hat{\mathbf{u}}_{K} \hat{\hat{\mathbf{s}}} \hat{\mathbf{v}}_{2}}+\frac{\pi}{\hat{\mathbf{s}}_{\mathbf{\mathbf { s }}} \hat{\mathbf{v}}_{2}}+\frac{\omega^{4}}{4} \int d^{2} \frac{\omega^{4}}{c_{q S V}^{5}(\hat{\mathbf{s}})}
\end{aligned}
$$

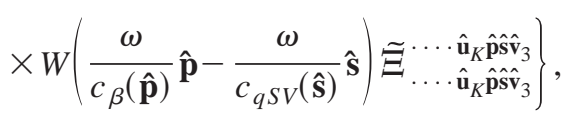

where $K$ is defined as the polarization for the wave type $\beta$ (1, 2 , or 3 for wave types $S H, q P$, and $q S V$, respectively). In Eq. (30), it can be seen that the integrals are over the unit sphere, which is defined by unit vector $\hat{\mathbf{s}}$. The direction $\hat{\mathbf{p}}$ defines the propagation direction, $\hat{\mathbf{s}}$ is the scattered direction, and $\hat{\mathbf{u}}$ and $\hat{\mathbf{v}}$ are defined as the polarization directions. The dependence of the vectors $\hat{\mathbf{u}}$ on $\hat{\mathbf{p}}$ and of $\hat{\mathbf{v}}$ on $\hat{\mathbf{s}}$ is implicit. The inner products on the covariance of the moduli fluctuations are given in terms of these four unit vectors. The argument of the correlation is the difference between the incoming and outgoing propagation directions.

It is clear that the above expressions of the attenuation for uniaxially aligned crack distributions are more complicated than those for a distribution of randomly oriented cracks as discussed previously. ${ }^{14}$ They can be simplified to the forms given there in the case of statistical isotropy. This framework is the same as that given by Turner. ${ }^{22}$ In the next section, the covariance and attenuation are specified.

\section{COVARIANCE AND ATTENUATION}

The relevant inner products on the covariance of the effective moduli fluctuations are necessary for calculating the attenuations. The tensorial part of the covariance is represented by an eighth-rank tensor which is given explicitly by

$$
\Xi(\mathbf{q}) \ldots \hat{\text { ûp } \hat{\mathbf{u}} \hat{\mathbf{p}} \hat{\mathbf{s}} \mathbf{v}}=\Xi(\mathbf{q})_{\alpha \beta \gamma \delta}^{i j m n} \hat{u}_{\beta} \hat{u}_{m} \hat{p}_{\alpha} \hat{p}_{n} \hat{s}_{i} \hat{s}_{\delta} \hat{v}_{\gamma} \hat{v}_{j} .
$$

For the case of uniaxially aligned cracks, the covariance is dependent on the crack orientations $\hat{\mathbf{m}}$. To calculate the covariance, the following identities are needed

$$
\begin{aligned}
& \left\langle\hat{m}_{i} \hat{m}_{j}\right\rangle=\frac{1}{2} \Delta_{i j}, \\
& \left\langle\hat{m}_{i} \hat{m}_{j} \hat{m}_{m} \hat{m}_{n}\right\rangle=\frac{1}{8}\left(\Delta_{i j} \Delta_{m n}+\Delta_{i m} \Delta_{j n}+\Delta_{i n} \Delta_{j m}\right), \\
& \left\langle\hat{m}_{i} \hat{m}_{j} \hat{m}_{m} \hat{m}_{n} \hat{m}_{\alpha} \hat{m}_{\beta}\right\rangle \\
& \quad=\frac{1}{48}\left[\Delta_{i j} \Delta_{m n} \Delta_{\alpha \beta}+\right.\text { all permutations } \\
& \quad-15 \text { terms in all }], \\
& \left\langle\hat{m}_{i} \hat{m}_{j} \hat{m}_{m} \hat{m}_{n} \hat{m}_{\alpha} \hat{m}_{\beta} \hat{m}_{\gamma} \hat{m}_{\delta}\right\rangle \\
& \quad=\frac{1}{384}\left[\Delta_{i j} \Delta_{m n} \Delta_{\alpha \beta} \Delta_{\gamma \delta}+\right.\text { all permutations } \\
& \quad-105 \text { terms in all }],
\end{aligned}
$$

where the brackets, \langle\rangle , denote an ensemble average, and $\Delta_{M N}=\left(\delta_{M N}-\hat{n}_{M} \hat{n}_{N}\right)$. The unit vector $\hat{\mathbf{n}}$ is the uniaxial symmetry axis. All averages of odd numbers of $\hat{\mathbf{m}}$ 's are zero.

In addition to these tensorial averages, the average of the spatial part of the covariance must be determined. As discussed previously, ${ }^{14}$ the necessary relation is given by $\langle H(\mathbf{x}) H(\mathbf{y})\rangle=\varepsilon \operatorname{Pr}(r \mid 0)$, where $\operatorname{Pr}(r \mid 0)=(1-\varepsilon) W(r)+\varepsilon$, is defined as the conditional probability. ${ }^{29}$ Due to the assumption of small damage density, the higher order terms may be neglected. Therefore, $\left\langle\delta C_{i j m n}(\mathbf{x}) \delta C_{\alpha \beta \gamma \delta}(\mathbf{y})\right\rangle$ $=\varepsilon W(r) \Xi_{i j m n}^{\alpha \beta \gamma \delta}$. Averaging over all crack orientations, the covariance is thus defined by

$$
\Xi_{i j m n}^{\alpha \beta \gamma \delta}=\frac{1}{2 \pi} \int_{0}^{2 \pi} \int_{-\pi / 2}^{\pi / 2}\left(\bar{C}_{i j m n}^{(s)} \bar{C}_{\alpha \beta \gamma \delta}^{(s)}\right) \delta\left(\varphi-\frac{\pi}{2}\right) \sin \varphi d \varphi d \theta,
$$

where the definition of $\overline{\mathbf{C}}^{(s)}$ is given by Eq. (60) in Ref. 14. Substituting the identities of Eqs. (32) into Eq. (33), the generally compact form of $\boldsymbol{\Xi}$ is constructed in terms of Kronecker deltas and pairs of $\hat{\mathbf{n}}$ ' $s$. The general compact form of $\Xi$ is not presented here due to brevity. The form of the attenuations given in Eqs. (30) is dependent on various inner products on the covariance tensor. The vectors $\hat{\mathbf{p}}$ and $\hat{\mathbf{s}}$, respectively, represent the incoming and outgoing propagation directions. The vectors $\hat{\mathbf{u}}$ and $\hat{\mathbf{v}}$ are vectors defining the polarization directions of the particular waves. These vectors are perpendicular to the plane defined by $\hat{\mathbf{s}}$ or $\hat{\mathbf{p}}$ and $\hat{\mathbf{n}}$ (for $S H$ waves) or they lie in this plane (for $q P$ and $q S V$ ).

Now the necessary inner products involved in determining the attenuations are calculated. The attenuations will vary angularly only within the plane defined by the propagation direction $\hat{\mathbf{p}}$ and the crack alignment direction $\hat{\mathbf{n}}$. Therefore, without loss of generality, a reference plane is defined as the $\hat{\mathbf{p}}$ - $\hat{\mathbf{n}}$ plane (see Fig. 3). The following vectors are then defined with respect to a general $x_{1} x_{2} x_{3}$ coordinate system as

$$
\begin{aligned}
& \hat{\mathbf{n}}=\hat{\mathbf{x}}_{3}, \quad \hat{\mathbf{p}}=\hat{\mathbf{x}}_{2} \sin \Theta+\hat{\mathbf{x}}_{3} \cos \Theta, \\
& \hat{\mathbf{s}}=\hat{\mathbf{x}}_{1} \sin \Theta^{\prime} \cos \phi^{\prime}+\hat{\mathbf{x}}_{2} \sin \Theta^{\prime} \sin \phi^{\prime}+\hat{\mathbf{x}}_{3} \cos \Theta^{\prime} .
\end{aligned}
$$

The polarization vectors are then defined with respect to these angles and $\psi$ as discussed by Turner. ${ }^{22}$ The angles $\gamma$ and $\gamma^{\prime}$ used hereafter are defined by

$$
\gamma=\Theta+\psi(\Theta), \quad \gamma^{\prime}=\Theta^{\prime}+\psi\left(\Theta^{\prime}\right) .
$$

These angles, $\gamma$ and $\gamma^{\prime}$, define the orientation angle of the $q P$ wave with respect to the $\hat{\mathbf{n}}$ direction, for the $\hat{\mathbf{p}}$ and $\hat{\mathbf{s}}$ directions, respectively.

Inserting these definitions of the relevant unit vectors into Eq. (33), the required inner products are reduced to a simple form. The inner products are:

$$
\begin{aligned}
& \text { for } \alpha_{S H} \\
& \Xi_{\cdots \cdots \hat{\mathbf{u}}_{1} \hat{\mathbf{p}} \hat{\mathbf{s}} \hat{\mathbf{v}}_{1}}^{\cdots}=\sin ^{2} \Theta \sin ^{2} \Theta^{\prime}\left[-\eta_{1} \cos ^{2} \phi^{\prime} \sin ^{2} \phi^{\prime}+\eta_{2}\right], \\
& \Xi_{\cdots \hat{\mathbf{u}}_{1} \hat{\mathbf{s}} \hat{\mathbf{s}} \hat{\mathbf{v}}_{2}}^{\cdots}=\sin ^{2} \Theta \sin ^{2} \Theta^{\prime} \sin ^{2} \gamma^{\prime}\left[\eta_{1} \cos ^{2} \phi^{\prime} \sin ^{2} \phi^{\prime}+\eta_{3}\right],
\end{aligned}
$$

$\Xi_{\cdots \hat{\mathbf{u}}_{1} \hat{\mathbf{p}} \hat{\mathbf{p}} \hat{\mathbf{v}}_{3}}^{\cdots}=\sin ^{2} \Theta \sin ^{2} \Theta^{\prime} \cos ^{2} \gamma^{\prime}\left[\eta_{1} \cos ^{2} \phi^{\prime} \sin ^{2} \phi^{\prime}+\eta_{3}\right]$, 


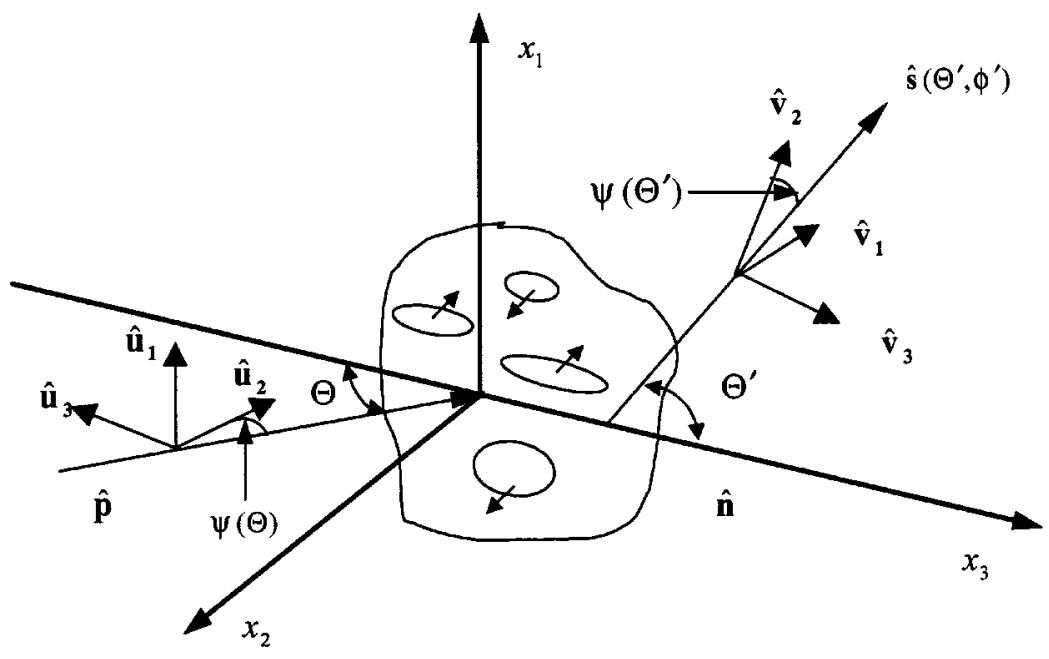

FIG. 3. Geometry for the propagation direction $\hat{\mathbf{p}}$, the scattered direction $\hat{\mathbf{s}}$, and the respective polarization directions $\hat{\mathbf{u}}$ and $\hat{\mathbf{v}}$ in the local coordinate system.

for $\alpha_{q P}$

$\Xi \cdots \hat{\mathbf{u}}_{\mathbf{2}} \hat{\mathbf{p}} \hat{\mathbf{s}} \hat{\mathbf{v}}_{\mathbf{1}}=\sin ^{2} \Theta \sin ^{2} \Theta^{\prime} \sin ^{2} \gamma\left[\eta_{1} \cos ^{2} \phi^{\prime} \sin ^{2} \phi^{\prime}+\eta_{3}\right]$,

$\Xi \cdots \hat{\mathbf{u}}_{2} \hat{\mathbf{p}} \hat{\mathbf{s}} \hat{\mathbf{v}}_{\mathbf{2}}=\sin ^{2} \Theta \sin ^{2} \Theta^{\prime} \sin ^{2} \gamma$

$$
\times \sin ^{2} \gamma^{\prime}\left[\eta_{1} \sin ^{4} \phi^{\prime}+\eta_{4} \sin ^{2} \phi^{\prime}+\eta_{5}\right],
$$

$\Xi \cdots \hat{\mathbf{u}}_{2} \hat{\mathbf{p}} \hat{\mathbf{s}} \hat{\mathbf{v}}_{\mathbf{3}}=\sin ^{2} \Theta \sin ^{2} \Theta^{\prime} \sin ^{2} \gamma$

$$
\times \cos ^{2} \gamma^{\prime}\left[\eta_{1} \sin ^{4} \phi^{\prime}+\eta_{4} \sin ^{2} \phi^{\prime}+\eta_{5}\right],
$$

and for $\alpha_{q S V}$

$\Xi \cdots \hat{\mathbf{u}}_{3} \hat{\mathbf{p}} \hat{\mathbf{s}} \hat{\mathbf{v}}_{\mathbf{1}}=\sin ^{2} \Theta \sin ^{2} \Theta^{\prime} \cos ^{2} \gamma\left[\eta_{1} \cos ^{2} \phi^{\prime} \sin ^{2} \phi^{\prime}+\eta_{3}\right]$,

$\exists \cdots \hat{\mathbf{u}}_{3} \hat{\mathbf{p}} \hat{\mathbf{s}} \hat{\mathbf{v}}_{\mathbf{2}}=\sin ^{2} \Theta \sin ^{2} \Theta^{\prime} \cos ^{2} \gamma$

$$
\times \sin ^{2} \gamma^{\prime}\left[\eta_{1} \sin ^{4} \phi^{\prime}+\eta_{4} \sin ^{2} \phi^{\prime}+\eta_{5}\right],
$$

$\Xi \cdots \hat{\mathbf{u}}_{3} \hat{\mathbf{p}} \hat{\mathbf{s}} \hat{\mathbf{v}}_{\mathbf{3}}=\sin ^{2} \Theta \sin ^{2} \Theta^{\prime} \cos ^{2} \gamma$

$$
\times \cos ^{2} \gamma^{\prime}\left[\eta_{1} \sin ^{4} \phi^{\prime}+\eta_{4} \sin ^{2} \phi^{\prime}+\eta_{5}\right],
$$

where $\gamma$ and $\gamma^{\prime}$ are defined in Eq. (35). The coefficients $\eta_{i}(i=1, \ldots, 5)$ and $T_{j}(j=1, \ldots, 7)$, are given by

$$
\begin{aligned}
& \eta_{1}=4 T_{3}+16 T_{6}+4 T_{7}, \quad \eta_{2}=T_{3}+4 T_{6}+4 T_{7}, \\
& \eta_{3}=T_{4}+4 T_{5}+4 T_{6}+6 T_{7}, \\
& \eta_{4}=4 T_{2}+4 T_{4}+32 T_{5}+16 T_{6}+16 T_{7}, \\
& \eta_{5}=T_{1}+4 T_{4}+4 T_{7},
\end{aligned}
$$

with

$$
\begin{aligned}
& T_{1}=-\frac{\nu^{2}\left(80 \nu^{4}-416 \nu^{3}+472 \nu^{2}+184 \nu-235\right) M^{2}}{128(1-2 \nu)^{2}}, \\
& T_{2}=-\frac{\nu\left(12 \nu^{3}-28 \nu^{2}+127 \nu-184\right) M^{2}}{384}, \\
& T_{7}=\frac{\nu^{2}(1-2 \nu)^{2} M^{2}}{384},
\end{aligned}
$$

$$
\begin{aligned}
& T_{3}=\frac{(\nu-4)(\nu-12)(1-2 \nu)^{2} M^{2}}{384}, \\
& T_{4}=\frac{\nu^{2}(10 \nu-23)(2 \nu-7) M^{2}}{384}, \\
& T_{5}=\frac{\nu(1-2 \nu)\left(6 \nu^{2}-31 \nu+44\right) M^{2}}{384}, \\
& T_{6}=\frac{(3-2 \nu)(1-2 \nu)^{2} M^{2}}{96},
\end{aligned}
$$

where the constant $M$ is defined as $M=\mu(32 / 3)[1-\nu /(2$ $-\nu)(1-2 \nu)]$. The expressions given by Eqs. (36)-(38) are also directly related to the diffuse energy propagation, including backscatter. ${ }^{30-32}$

If the tensorial and spatial components of covariance are assumed to be independent, as discussed in Eq. (17), the spatial correlation function $W$ is uncorrelated with the tensorial part. Here, the correlation function $W$ is assumed to have an exponential form $W(r)=e^{-r / L}$, where $L$ is the spatial correlation length, $L=2\langle a\rangle$. The limits of such an assumption have been reviewed by Stanke, ${ }^{33}$ and Markov and Willis. $^{29}$

Substituting the above inner products into Eqs. (30) and integrating over the azimuthal angle $\phi^{\prime}$, the attenuations finally reduce to dimensionless forms

$$
\begin{aligned}
\alpha_{S H}(\Theta) L= & x_{S H}^{4} \frac{\varepsilon}{2 \rho^{2} \bar{c}_{S H}^{4}} r_{S H}^{3}(\Theta) \sin ^{2} \Theta\left[I_{S H-S H}\right. \\
& \left.+I_{S H-q P}\left(\frac{\bar{c}_{S H}}{\bar{c}_{q P}}\right)^{5}+I_{S H-q S V}\left(\frac{\bar{c}_{S H}}{\bar{c}_{q S V}}\right)^{5}\right], \\
\alpha_{q P}(\Theta) L= & x_{q P}^{4} \frac{\varepsilon}{2 \rho^{2} \bar{c}_{q P}^{4}} r_{q P}^{3}(\Theta) \sin ^{2} \Theta \sin ^{2} \gamma \\
& \times\left[I_{q P-S H}\left(\frac{\bar{c}_{q P}}{\bar{c}_{S H}}\right)^{5}+I_{q P-q P}+I_{q P-q S V}\left(\frac{\bar{c}_{q P}}{\bar{c}_{q S V}}\right)^{5}\right],
\end{aligned}
$$




$$
\begin{aligned}
\alpha_{q S V}(\Theta) L= & x_{q S V}^{4} \frac{\varepsilon}{2 \rho^{2} \bar{c}_{q S V}^{4}} r_{q S V}^{3}(\Theta) \sin ^{2} \Theta \cos ^{2} \gamma \\
& \times\left[I_{q S V-S H}\left(\frac{\bar{c}_{q S V}}{\bar{c}_{S H}}\right)^{5}+I_{q S V-q P}\left(\frac{\bar{c}_{q S V}}{\bar{c}_{q P}}\right)^{5}\right. \\
& \left.+I_{q S V-q S V}\right],
\end{aligned}
$$

with the density, $\rho$, now included in the general form. The terms denoted by $I_{\beta-\gamma}$ within the square brackets represent integrals defined by

$$
\begin{aligned}
I_{S H-S H}= & \int_{0}^{\pi}\left[\frac{\eta_{1}\left(6 X_{S H-S H}^{2}-Y_{S H-S H}^{2}\right)}{2 Y_{S H-S H}^{2}}\right. \\
& +\frac{\eta_{1}\left(2 X_{S H-S H} Y_{S H-S H}^{2}-3 X_{S H-S H}^{2}\right)}{Y_{S H-S H}^{4}\left(X_{S H-S H}^{2}-Y_{S H-S H}^{2}\right)^{1 / 2}} \\
& \left.\times \frac{\eta_{2} X_{S H-S H}}{\left(X_{S H-S H}^{2}-Y_{S H-S H}^{2}\right)^{3 / 2}}\right] r_{S H}^{5}\left(\Theta^{\prime}\right) \sin ^{3} \Theta^{\prime} d \Theta^{\prime},
\end{aligned}
$$

$$
\begin{aligned}
I_{S H-\alpha}= & \int_{0}^{\pi}\left[\frac{\eta_{1}\left(Y_{S H-\alpha}^{2}-6 X_{S H-\alpha}^{2}\right)}{2 Y_{S H-\alpha}^{2}}\right. \\
& +\frac{\eta_{1}\left(3 X_{S H-\alpha}^{2}-2 X_{S H-\alpha} Y_{S H-\alpha}^{2}\right)}{Y_{S H-\alpha}^{4}\left(X_{S H-\alpha}^{2}-Y_{S H-\alpha}^{2}\right)^{1 / 2}} \\
& \left.\times \frac{\eta_{3} X_{S H-\alpha}}{\left(X_{S H-\alpha}^{2}-Y_{S H-\alpha}^{2}\right)^{3 / 2}}\right] r_{\alpha}^{5}\left(\Theta^{\prime}\right) \Pi_{\alpha} \sin ^{3} \Theta^{\prime} d \Theta^{\prime},
\end{aligned}
$$

and

$$
\begin{aligned}
I_{\alpha-S H}= & \int_{0}^{\pi}\left[\frac{\eta_{1}\left(Y_{\alpha-S H}^{2}-6 X_{\alpha-S H}^{2}\right)}{2 Y_{\alpha-S H}^{2}}\right. \\
& +\frac{\eta_{1}\left(3 X_{\alpha-S H}^{2}-2 X_{\alpha-S H} Y_{\alpha-S H}^{2}\right)}{Y_{\alpha-S H}^{4}\left(X_{\alpha-S H}^{2}-Y_{\alpha-S H}^{2}\right)^{1 / 2}} \\
& \left.\times \frac{\eta_{2} X_{\alpha-S H}}{\left(X_{\alpha-S H}^{2}-Y_{\alpha-S H}^{2}\right)^{3 / 2}}\right] r_{S H}^{5}\left(\Theta^{\prime}\right) \sin ^{3} \Theta^{\prime} d \Theta^{\prime},
\end{aligned}
$$

$$
\begin{aligned}
I_{\delta-\alpha}= & \int_{0}^{\pi}\left[\frac{\eta_{1}\left(6 X_{\delta-\alpha}^{2}+Y_{\delta-\alpha}^{2}\right)+2 \eta_{4} Y_{\delta-\alpha}^{2}}{2 Y_{\delta-\alpha}^{4}}+\frac{\eta_{1}\left(4 X_{\delta-\alpha}^{3} Y_{\delta-\alpha}^{2}-3 X_{\delta-\alpha}^{5}\right)+\eta_{4} Y_{\delta-\alpha}^{2}\left(2 X_{\delta-\alpha} Y_{\delta-\alpha}^{2}-X_{\delta-\alpha}^{3}\right)}{Y_{\delta-\alpha}^{4}\left(X_{\delta-\alpha}^{2}-Y_{\delta-\alpha}^{2}\right)^{3 / 2}}\right. \\
& \left.+\frac{\eta_{5} X_{\delta-\alpha} Y_{\delta-\alpha}^{4}}{Y_{\delta-\alpha}^{4}\left(X_{\delta-\alpha}^{2}-Y_{\delta-\alpha}^{2}\right)^{3 / 2}}\right] r_{\alpha}^{5}\left(\Theta^{\prime}\right) \Pi_{\alpha} \sin ^{3} \Theta^{\prime} d \Theta^{\prime}
\end{aligned}
$$

with

$$
\begin{aligned}
X_{\beta-\gamma}= & 1+x_{\beta}^{2} r_{\beta}^{2}(\Theta)+x_{\gamma}^{2} r_{\gamma}^{2}\left(\Theta^{\prime}\right) \\
& -2 x_{\beta} x_{\gamma} r_{\beta}(\Theta) r_{\gamma}\left(\Theta^{\prime}\right) \cos \Theta \cos \Theta^{\prime}, \\
Y_{\beta-\gamma}= & 2 x_{\beta} x_{\gamma} r_{\beta}(\Theta) r_{\gamma}\left(\Theta^{\prime}\right) \sin \Theta \sin \Theta^{\prime},
\end{aligned}
$$

for the different wave types, $\beta$ and $\gamma$. The subscripts $\delta$ and $\alpha$ denote either the $q P$ or $q S V$ wave type, and the notation $\Pi_{q P}=\sin ^{2}\left(\Theta^{\prime}+\psi\left(\Theta^{\prime}\right)\right), \Pi_{q S V}=\cos ^{2}\left(\Theta^{\prime}+\psi\left(\Theta^{\prime}\right)\right)$ is used. In Eqs. (41)-(46), the angular averaged wave speeds are defined as $\bar{c}_{\beta}=\frac{1}{2} \int_{0}^{\pi} c_{\beta}(\Theta) \sin \Theta d \Theta$, for each wave type, $\beta$. Three nondimensional frequencies are then defined as $x_{\beta}$ $=\omega L / \bar{c}_{\beta}$ and the slowness surface for each wave type is defined by the dimensionless quantity $r_{\beta}(\Theta)=\bar{c}_{\beta} / c_{\beta}(\Theta)$. Equations $(41)-(45)$ are the primary results of this article.

In the long wavelength Rayleigh limit, $x_{\beta} \ll 1$ and these integrals become independent of incident direction and frequency. Therefore, they reduce to a much simpler form as

$$
\begin{aligned}
& I_{S H-S H}=\int_{0}^{\pi}\left(-\frac{\eta_{1}}{8}+\eta_{2}\right) r_{S H}^{5}\left(\Theta^{\prime}\right) \sin ^{3} \Theta^{\prime} d \Theta^{\prime}, \\
& I_{S H-\alpha}=\int_{0}^{\pi}\left(\frac{\eta_{1}}{8}+\eta_{3}\right) r_{\alpha}^{5}\left(\Theta^{\prime}\right) \Pi_{\alpha} \sin ^{3} \Theta^{\prime} d \Theta^{\prime},
\end{aligned}
$$

and

$$
\begin{aligned}
& I_{\alpha-S H}=\int_{0}^{\pi}\left(\frac{\eta_{1}}{8}+\eta_{3}\right) r_{S H}^{5}\left(\Theta^{\prime}\right) \sin ^{3} \Theta^{\prime} d \Theta^{\prime}, \\
& I_{\delta-\alpha}=\int_{0}^{\pi}\left(\frac{3 \eta_{1}}{8}+\frac{\eta_{4}}{2}+\eta_{5}\right) r_{\alpha}^{5}\left(\Theta^{\prime}\right) \Pi_{\alpha} \sin ^{3} \Theta^{\prime} d \Theta^{\prime}
\end{aligned}
$$

for all outgoing wave types. In the Rayleigh limit, the angular dependence of the attenuation is explicitly seen. In the subsequent section, example numerical results and discussion are presented.

\section{EXAMPLE RESULTS}

Numerical results are now presented for a specific case, in which the observed anisotropy of the cracked material is essentially due to the presence of the uniaxially aligned cracks. The material properties of the uncracked medium used are Young's modulus $E=2.0 \times 10^{11} \mathrm{~Pa}$, Poisson's ratio $\nu=0.30$, and density $\rho=7850 \mathrm{~kg} / \mathrm{m}^{3}$. Using the dispersion relations given in Eqs. (25), the slowness surfaces calculated for different damage densities, $\varepsilon=0$ and 0.1 , are shown in Fig. 4 . The normalized effective wave velocity, $c_{\beta}(\varepsilon) / c_{\beta}(\varepsilon$ $=0$ ), of each wave type is presented in Fig. 5. The effective velocities decrease with increasing damage density $\varepsilon$ within the considered frequency range. The reduction of velocity of $S H$ and $q P$ wave due to the presence of the uniaxially aligned cracks is a maximum at $\Theta=90^{\circ}$, it becomes smaller as $\Theta$ decreases, and the reduction reaches a minimum at $\Theta$ 


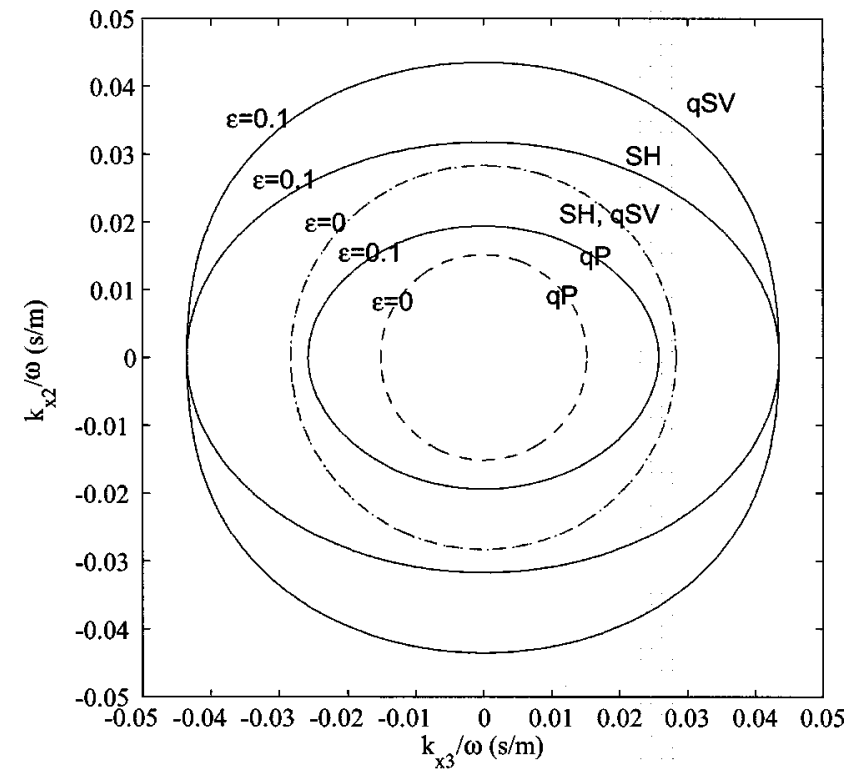

FIG. 4. Slowness surfaces for damage densities $\varepsilon=0,0.1$.

$=0^{\circ}$, though the changes are not substantial. These results are in basic agreement with those of Zhang and Gross ${ }^{10}$ and Eriksson and Datta. ${ }^{13}$ The $q S V$ wave velocity is seen to have a greater reduction at $\Theta=45^{\circ}$ than at $\Theta=0^{\circ}$ and $90^{\circ}$.

In the Rayleigh limit, the attenuations simplify considerably since the integrals reduce to those given by Eqs. (47) and (48). The attenuation depends on the fourth power of frequency in the Rayleigh regime. Thus, the angular Rayleigh attenuation results shown in Fig. 6 are given in a general form of, $\alpha L /\left(x^{4} \varepsilon\right)$, for each wave type. In Fig. 6, the $S H$ and $q P$ waves are observed to have their maxima at $\Theta$ $=90^{\circ}$ - perpendicular to the crack alignment direction $\hat{\mathbf{n}}$. The $q S V$ wave is observed to have zero attenuation for propagation along the symmetry axis $\left(\Theta=0^{\circ}\right)$ and perpendicular to it $\left(\Theta=90^{\circ}\right)$. All wave types have zero attenuation along the symmetry axis, because the material properties do not vary in that direction. Those results are qualitatively the same as previous work. ${ }^{19,21,22}$ Zhang and Gross ${ }^{10}$ comment

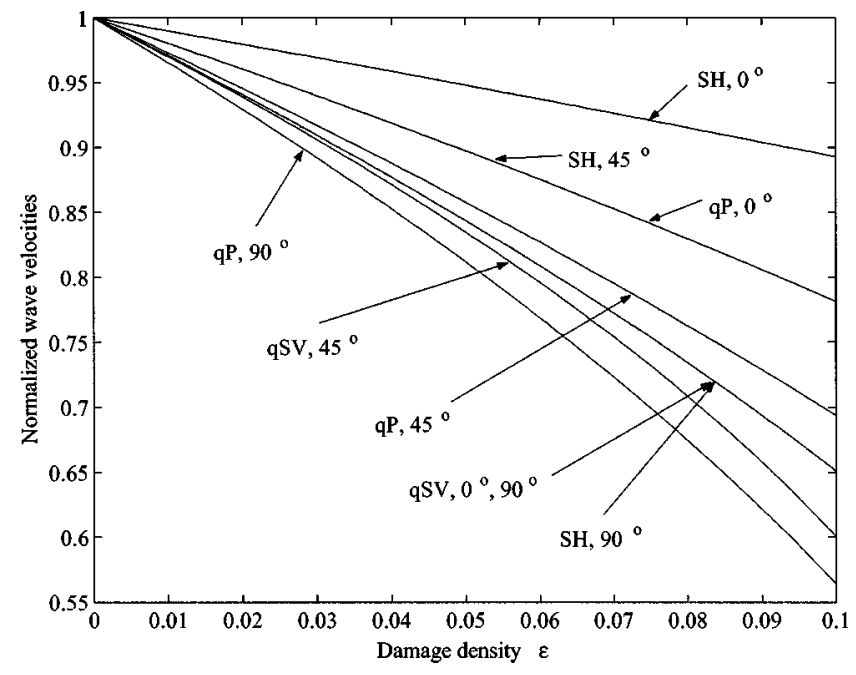

FIG. 5. Wave velocity of each wave type normalized to the undamaged wave speed, $c_{\beta}(\varepsilon) / c_{\beta}$, versus damage density $\varepsilon$ at $\Theta=0^{\circ}, 45^{\circ}$ and $90^{\circ}$.

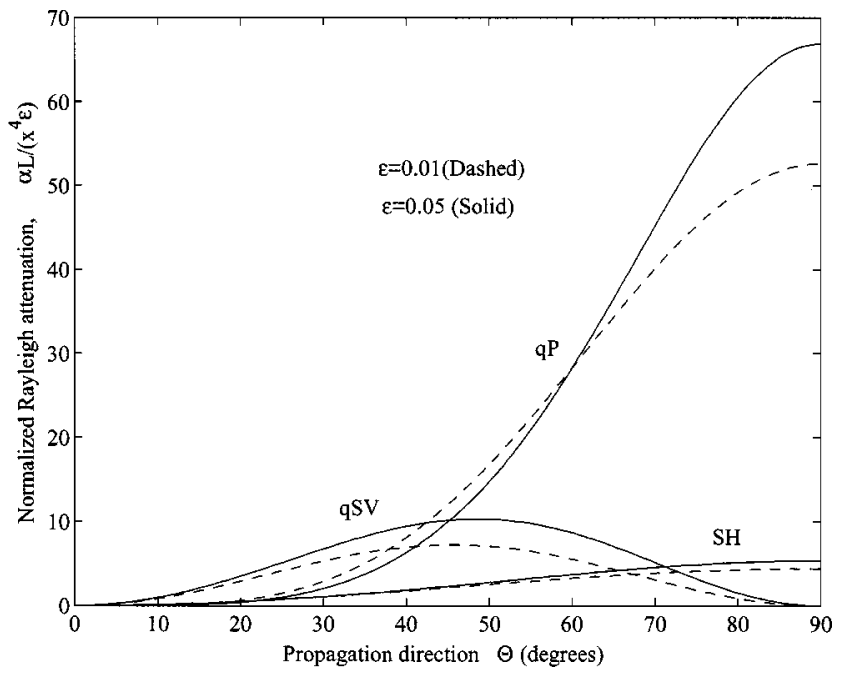

FIG. 6. Rayleigh limit as a function of direction for the $S H, q P$, and $q S V$ waves for damage density $\varepsilon=0.01$ (dashed) and $\varepsilon=0.05$ (solid). The dimensionless attenuation $\alpha L$ has been normalized by the fourth power of the dimensionless frequency and damage density for the respective wave type: $\alpha_{S H} L /\left(x_{S H}^{4} \varepsilon\right), \alpha_{q P} L /\left(x_{q P}^{4} \varepsilon\right)$, and $\alpha_{q S V} L /\left(x_{q S V}^{4} \varepsilon\right)$.

that their attenuation results are not zero for propagation along the symmetry axis. They speculate that the attenuation arises from Poisson effects. However, such a comparison is difficult to make since the focus of their work was at much higher frequencies. An additional feature observed for the $q S V$ wave in Fig. 6, is the asymmetry that develops as $\varepsilon$ increases. This peak is around $\Theta=45^{\circ}$, but shifts slightly as $\varepsilon$ increases from 0.01 to 0.05 .

Finally, using Eqs. (41)-(43), attenuation results are given in terms of the single dimensionless frequency $x_{S H}$ $=\omega L / \bar{c}_{S H}$. Outside the Rayleigh regime, the attenuations were calculated using the complete integrals, Eqs. (44) and (45), by numerical integration. In Fig. 7, the normalized $S H$ wave attenuation, $\alpha_{S H} / k_{S H}$, is presented as a function of propagation direction for three different damage densities at frequency $x_{S H}=1.0$. The attenuation for propagation perpendicular to the crack alignment direction is seen to increase more quickly than for other directions as the damage in-

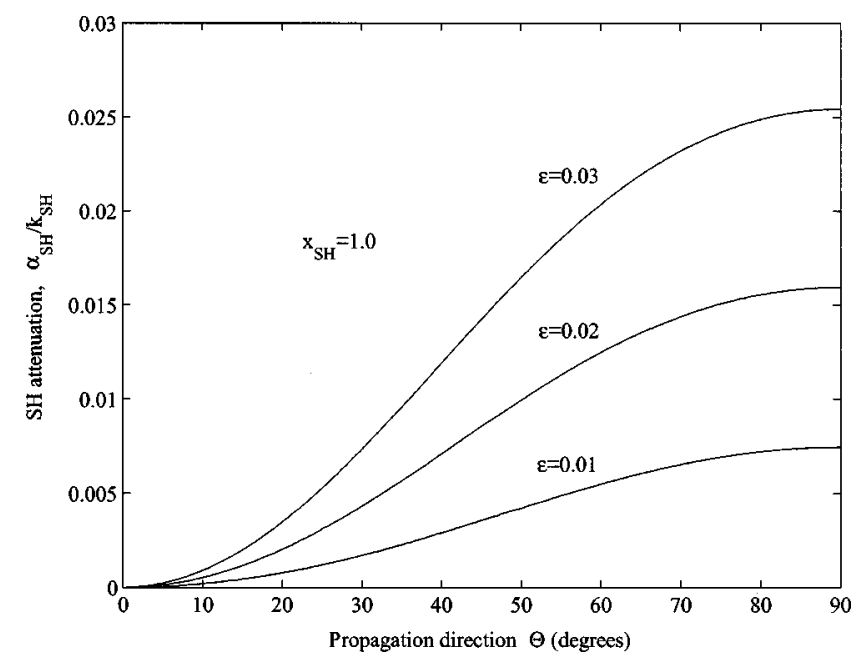

FIG. 7. Angular dependence of the normalized $S H$ attenuation, $\alpha_{S H} / k_{S H}$ for various damage densities $\varepsilon$ at frequency $x_{S H}=1.0$. 


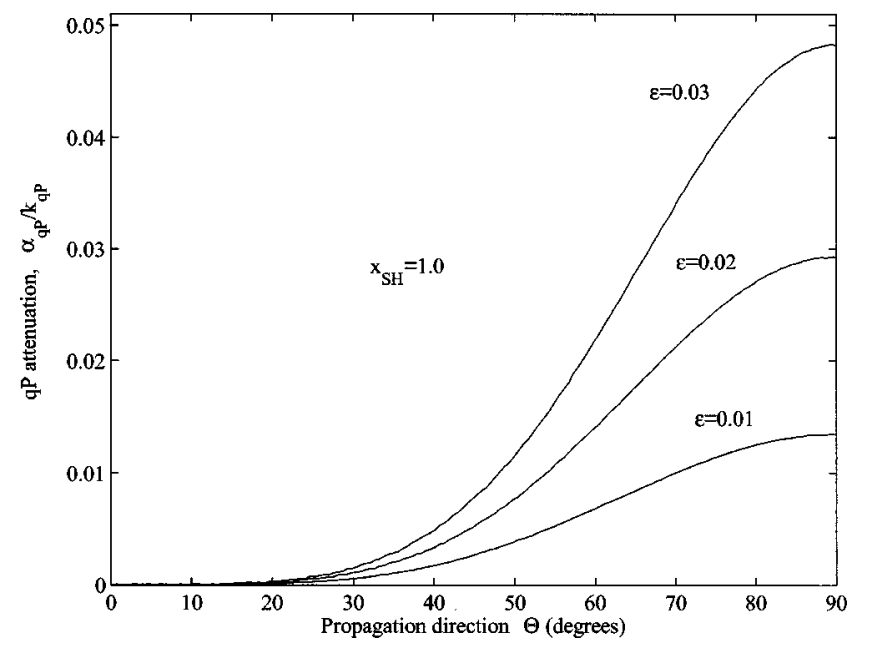

FIG. 8. Angular dependence of the normalized $q P$ attenuation, $\alpha_{q P} / k_{q P}$ for various damage densities $\varepsilon$ at frequency $x_{S H}=1.0$.

creases. The results for the normalized $q P$ attenuation, $\alpha_{q P} / k_{q P}$, are shown in Fig. 8. These results display similar behavior as the $S H$ attenuation in terms of the change with angle and damage. Analogous results have been observed in textured polycrystals by Hirsekorn, ${ }^{21}$ Ahmed and Thompson, ${ }^{19}$ and Turner. $^{22}$ In Fig. 9, the normalized $q S V$ attenuation, $\alpha_{q S V} / k_{q S V}$, is presented at various damage densities for frequency $x_{S H}=1.0$. The attenuation for propagation at $\Theta=0^{\circ}$ and $90^{\circ}$ is zero as discussed above. For propagation at $\Theta=45^{\circ}$, the attenuation is the largest. In addition, it is seen that the peak of maximum attenuation shifts as the damage increases, although this shift is not significant. The direction of maximum $\alpha_{q S V}$ is dependent upon both frequency and damage. This shift is thought to be the result of the induced anisotropy from the cracks as shown in the slowness plots in Fig. 4 as speculated elsewhere. ${ }^{22}$ However, further investigation is necessary to determine the precise reason for this peak shift.

\section{CONCLUSIONS}

In this article, elastic wave propagation and scattering have been examined for media with uniaxially aligned

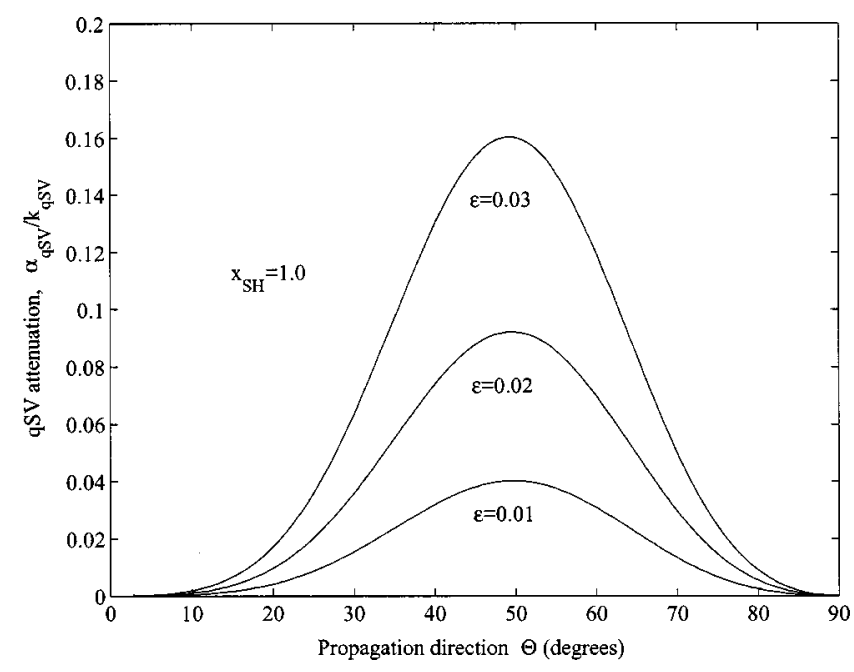

FIG. 9. Angular dependence of the normalized $q S V$ attenuation, $\alpha_{q S V} / k_{q S V}$ for various damage densities $\varepsilon$ at frequency $x_{S H}=1.0$. cracks. These cracks have unit normals that are randomly oriented within a plane of isotropy. The ensemble average elastic wave response is governed by the Dyson equation which is solved within the limits of the first-order smoothing approximation. The general Green's dyadic for a transversely isotropic medium was employed to derive expressions of the attenuation of the shear horizontal, quasilongitudinal and quasishear vertical waves. This dyadic approach is convenient to make the results coordinate free. Thus, the final forms of the attenuations for the three wave types were given directly by simple compact expressions involving integrations over the unit circle. In particular, the integrals are simplified considerably in the Rayleigh regime. The general attenuations for each wave type are dependent on frequency, wave velocity, wave direction and damage density. Finally, numerical results show how the attenuations and the effective wave velocity of each wave type are affected by those parameters. The general formulation is also directly related to other types of elastic wave scattering such as backscatter. The simple form of the results makes them particularly useful for nondestructive testing and materials characterization research. However, the neglect of mutual interactions among the microcracks may have a large influence for the scattering effects. This analysis will be investigated in subsequent work.

\section{ACKNOWLEDGMENTS}

The National Science Foundation (Grants No. CMS9978707 and INT-0089548) and the National Bridge Research Organization (NaBRO) are gratefully acknowledged by the authors for supporting this work.

${ }^{1}$ A. K. Mal, "Interaction of elastic waves with a penny-shaped crack," Int. J. Eng. Sci. 8, 381-388 (1968).

${ }^{2}$ A. K. Mal, "A note on the low frequency diffraction of elastic waves by a Griffith crack,” Int. J. Eng. Sci. 10, 609-612 (1972).

${ }^{3}$ M. Piau, "Attenuation of a plane compressional wave by a random distribution of thin circular cracks," Int. J. Eng. Sci. 17, 151-167 (1979).

${ }^{4}$ A. Chatterjee, A. K. Mal, L. Knopoff, and J. Hudson, "Attenuation of elastic waves in a cracked, fluid-saturated solid," Math. Proc. Cambridge Philos. Soc. 88, 547-561 (1980).

${ }^{5}$ J. A. Hudson, "Wave speeds and attenuation of elastic waves in material containing cracks," Geophys. J. R. Astron. Soc. 64, 133-150 (1981).

${ }^{6}$ P. A. Martin, "Diffraction of elastic waves by a penny-shaped crack," Proc. R. Soc. London, Ser. A 378, 263-285 (1981).

${ }^{7}$ S. Krenk and H. Schmidt, "Elastic wave scattering by a circular crack," Philos. Trans. R. Soc. London, Ser. A 308, 167-198 (1982).

${ }^{8}$ P. A. Martin and G. R. Wickham, "Diffraction of elastic waves by a penny-shaped crack: Analytical and numerical results," Proc. R. Soc. London, Ser. A 390, 91-129 (1983).

${ }^{9}$ C. Zhang and J. Achenbach, "Effective wave velocity and attenuation in a material with distributed penny-shaped cracks," Int. J. Solids Struct. 27, $751-767$ (1991)

${ }^{10}$ C. Zhang and D. Gross, "Wave attenuation and dispersion in randomly cracked solids-I. Slit cracks,” Int. J. Eng. Sci. 31, 841-858 (1993).

${ }^{11}$ C. Zhang and D. Gross, "Wave attenuation and dispersion in randomly cracked solids-II. Penny-shaped cracks," Int. J. Eng. Sci. 31, 859-872 (1993).

${ }^{12}$ V. P. Smyshlyaev and J. R. Willis, "Linear and nonlinear scattering of elastic waves by microcracks,” J. Mech. Phys. Solids 42, 585-610 (1994).

${ }^{13}$ A. S. Eriksson, A. Bostrom, and S. K. Datta, "Ultrasonic wave propagation through a cracked solid," Wave Motion 22, 297-310 (1995).

${ }^{14}$ L. Yang and J. A. Turner, "Scattering of elastic waves in damaged media," J. Acoust. Soc. Am. 113, 2992-3000 (2003).

${ }^{15}$ S. Nemat-Nasser and M. Hori, Micromechanics: Overall Properties of 
Heterogeneous Materials (North-Holland, Amsterdam, 1993).

${ }^{16}$ M. Kachanov, "Elastic solids with many cracks and related problems,", Adv. Appl. Mech. 29, 259-445 (1993).

${ }^{17}$ D. Krajcinovic, Damage Mechanics (North-Holland, Amsterdam, 1996).

${ }^{18}$ F. E. Stanke and G. S. Kino, "A unified theory for elastic wave propagation in polycrystalline materials," J. Acoust. Soc. Am. 75, 665-681 (1984).

${ }^{19} \mathrm{~S}$. Ahmed and R. B. Thompson, "Propagation of elastic waves in equiaxed stainless-steel polycrystals with aligned [001] axes," J. Acoust. Soc. Am. 99, 2086-2096 (1996).

${ }^{20} \mathrm{~S}$. Hirsekorn, "The scattering of ultrasonic waves in polycrystalline materials with texture," J. Acoust. Soc. Am. 77, 832-843 (1985).

${ }^{21} \mathrm{~S}$. Hirsekorn, "Directional dependence of ultrasonic propagation in textured polycrystals," J. Acoust. Soc. Am. 79, 1269-1279 (1986).

${ }^{22}$ J. A. Turner, "Elastic wave propagation and scattering in heterogeneous, anisotropic media: Textured polycrystalline materials," J. Acoust. Soc. Am. 106, 541-552 (1999).

${ }^{23}$ U. Frisch, "Wave propagation in random media," in Probabilistic Methods in Applied Mathematics, edited by A. T. Barucha-Reid (Academic, New York, 1968), Vol. 1, pp. 75-198.

${ }^{24}$ R. L. Weaver, "Diffusion of ultrasound in polycrystals," J. Mech. Phys. Solids 38, 55-86 (1990).

${ }^{25}$ F. C. Karal and J. B. Keller, "Elastic, electromagnetic, and other waves in a random medium,” J. Math. Phys. 5, 537-547 (1964).

${ }^{26}$ I. Kunin, Elastic Media with Microstructure II (Springer-Verlag, Berlin, 1983).

${ }^{27}$ J. Walsh, "The effect of cracks on compressibility of rocks," J. Geophys. Res. 70, 381-389 (1965).

${ }^{28}$ B. Budiansky and R. J. O'Connell, "Elastic moduli of a cracked solid," Int. J. Solids Struct. 12, 81-97 (1976).

${ }^{29}$ K. Z. Markov and J. R. Willis, "On the two-point correlation function for dispersions of nonoverlapping spheres," Math. Methods Appl. Sci. 8, 359-377 (1998)

${ }^{30}$ J. A. Turner and R. L. Weaver, "Radiative transfer and multiple scattering of diffuse ultrasound in polycrystalline media," J. Acoust. Soc. Am. 96, 3675-3683 (1994).

${ }^{31}$ J. A. Turner and R. L. Weaver, "Time dependence of multiply scattered diffuse ultrasound in polycrystalline media," J. Acoust. Soc. Am. 97, 2639-2644 (1995).

${ }^{32}$ J. A. Turner and R. L. Weaver, "Ultrasonic radiative transfer theory: Effects of a fluid-solid interface," J. Acoust. Soc. Am. 98, 2801-2808 (1995).

${ }^{33}$ F. E. Stanke, "Spatial autocorrelation functions for calculations of effective propagation constants in polycrystalline materials," J. Acoust. Soc. Am. 80, 1479-1485 (1986). 